\title{
A Systematic Literature Review of Blockchain-Based Crops Traceability in Agricultural Supply Chain
}

\author{
${ }^{1}$ S M Nazmuz Sakib (Orchid- https://orcid.org/0000-0001-9310-3014) (sakibpedia@gmail.com) \\ ${ }^{1}$ Graduate of BSc in Business Studies \\ School of Business And Trade \\ Pilatusstrasse 6003, 6003 Luzern, Switzerland \\ ${ }^{1}$ Student of LLB(Hon's) \\ Department of Law \\ Dhaka International University \\ House \# 4, Road \# 1, Block - F, Dhaka 1213
}

\begin{abstract}
:
In recent years, Blockchain has been favorably adopted in the Supply Chain industry as it provides guaranteed transparency and traceability. The flexibility of Blockchain allows different applications to enable to exchange information; a significant middleweight layer is responsible for information transfer in the Agricultural Sector. The products that are manufactured at a global level in the agriculture industry are improved in safety, validation of some criteria, and quality. In the agriculture industry, the increasing number of complications associated with food safety and impurity risks needs high-level effective traceability solutions that act as necessary quality management tools to make sure satisfactory safety of crops. The agricultural supply chain today has complex ecosystems, consisting of several stockholders to authenticate criteria which are important like crop development stages, monitoring and validation, and compliance with the quality standard. In this proposed research, a systematic literature review will be done that includes smart contracts, Blockchain, and business transactions exclusively for crop production traceability across the agricultural and food supply chain. By using Blockchain in the agriculture sector, productivity, consistency, safety, reliability, and advanced security are increased. All transactions are kept and recorded on the immutable record of Blockchain associates with a decentralized system. Thus, it provides more traceability and clarity in the agriculture system in a

safe, trustworthy, and effective way. A systematic literature review is thus enforced to classify the papers which are selected by the following classification: crop traceability, contribution type, research type, and their approach. For this systematic review, the papers which extracted are
\end{abstract}


classified according to defined criteria. The purpose of this study is to fill the gap by collecting and analyzing studies available within the literature aiming to firstly, gain complete insight on the integration of Blockchain in the agriculture sector. Secondly, provide a summary of the present state of research on this area and identify gaps in existing studies. To achieve this aim an SLR was conducted. The findings of this SLR are discussed and researchers were provided with suggestions on possible directions for future research.

Keywords: Blockchain, Agriculture, Traceability, Food Supply chain, Crops.

\section{Introduction:}

Blockchain may be a large transaction book, retained by a diverse number of computers which does not rely on any third party. Transactions or data files are managed through separate platforms that permit the information is communicated, managed, kept, and displayed in an understandable format (Batubara F. et. al., 2018). Blockchain could be used to protect an auto trading mechanism. Blockchain develops a safe and secure food dealing system. The Blockchain association may be a separate authorization block with multiple authorized notes to start the shared ledger with moderate value (Lin Y P et. al., 2017).

The food supply chain system is an important part of food trade. There are many stakeholders in the food trade of agriculturalists, processors, dealers, traders, retailers, and consumers. Some organizations have created provisions to make a good trading environment. (Mao D. et. al., 2019). For example, stop an aggravated unfair trading environment within the food supply chain to assure fair treatment and farming trades. These include late payments, food crops, last-minute order cancellations, and unilateral or retrospective changes to agreements (Mao D. et. al., 2019). These issues will not only reduce the efficiency but will also undermine the stability of the food market because the difficulty of the transaction process will lead to high transaction prices and long transaction periods which can lead to inefficient transactions. Therefore, it is necessary to seek 
clarifications that can protect transaction balance and promote food market productivity. (Mao D. et. al., 2019).

Product traceability in agriculture needs to gather, communicate, and manage vital information through the initiation of the initiative, the exchange of various information within the supply chain. (Tian F., 2010). Blockchain will bring sensible, realistic, and significant solutions to make sure the agriculture products traceability which removes the requirement of a trusted third party authority (Tian F., 2010). The overarching impartiality to show that Blockchain and Ethereum agreements will track and changeless integration of business contracts within the agriculture system. We usually examine Blockchain perimeter to offer traceability and reflectivity within crop supply chains (Salah K et. al., 2019).

The agriculture system is the policy, planning, execution, and monitoring of activities to source good infrastructure, coordinate supply that supplies demand, and measure performance globally (Caro M P et. al., 2018). Smart contracts are the program that is executed automatically certain processes and saves the result in Blockchain in a highly reliable way. However, these contracts and applications have a huge impact in a variety of areas, especially in industries that have a major impact on the Blockchain(Caro M. et. al., 2018). Different types of new distribution applications were implemented based on Blockchain. Some of these applications focus on automation and process conversion within the economic sector. Automated processes save money and increase transparency (Kamilaris A et. Al., 2019).

Blockchain association is known on licensed bulges to publicly examine and share dealership history and not rely on a definite third party. Food trade history among user bulges is uploaded to the licensed bulbs after encryption. The authorized nodes will be auditing the transactions and recording them within the shared ledger. This ledger is accessible to the general public through nodes connected to the Blockchain Association (Lin Y P et. al., 2017). The operations of the agriculture supply chain are vast or multifaceted with many stakeholders from processors, agriculturalists, consumers, and constructors. The agriculture system is getting a lot of importance nowadays because the old system is complex and time-consuming and it's very difficult to track back the product base (Salah K et. al., 2019). 
The Blockchain can potentially be used to greatly enhance efficiency and thus Swiss agricultural competitiveness for example for inspections is often automatic, and therefore results are often held securely and in a manner distribution (Kamilaris A. et. al, 2019). All transactions are distributed through the machine network that runs the Blockchain procedure and should be valid at all computation bulges(Lin W. et al, 2020). Maintain a trusted view is the key component of Blockchain and agreement between its members. Although some may not be honest. Researchers have extensively studied the contents of the agreement in the past, however, this focused on new impetus given in Blockchain. And new proposals as a result of Block systems (Kamilaris A. et. al, 2019).

In theory, communications technology can be a centralized force but information technology can be a decentralized force. However, it can be a powerful tool to include broadband network structure, wireless element detection technologies, and mobile devices that are helpful in information gathering, assortment, authentication, investigate, and communicate. Since the ICT system allows different shareholders (Lin Y P et. al., 2017). In Agriculture, it is necessary to keep trust and consistency throughout the entire supply chain. It is too necessary that the records stored may be tamper-proof and the transactions transmitted without relying on any third-party centralized intermediaries. The Blockchain is an achievable resolution to alleviate these problems and considerations (Caro M. et. al., 2018). In agriculture administrator, a huge amount of traditional logistics information is to be stored and the correct arrangement of orders and deliveries and does not provide features like traceability, clarity, auditing. By using these features we improve food quality(Motta G.A et,al 2020). This is why various Development analytics departments focusing on their efforts on adopting different internet technologies (Kamilaris A. et. al, 2019).

Agricultural product development and observation and the effective management of supply in agriculture and food provide a chain area unit necessary to form positive product safety. The growing issues of food security and pollution risks are reviewing the main focus of improved traceability across the supply chain (Tian F., 2010). Currently while in recent years it is developed rapidly that the agricultural food supply chain systems area unit is still in its prime phase, and its area units still face several issues, such as equipment shortages and modems, low levels of information. Enforced systems, disorderly regulation, and lack of monitoring of traceability 
systems. For these reasons, food safety events are usually broken out, creating customers with vulnerable groups within the markets (Tian F., 2010).

\section{Research Questions:}

The purpose of SMS is to raise awareness and provide solutions to handle agriculture issues to gain a detailed insight into this issue and this systematic mapping study addresses these questions. These questions will enable us to classify existing Blockchain research by use in agriculture and identify future areas of research in the field. These are the questions.

1. By using Blockchain on a global level how to reduce the risk factor of quality, validation, and safety in the agriculture sector?

2. How to improve agriculture crops and foods with Blockchain?

3. How we can use Blockchain in the agriculture sector without financial intermediaries?

\section{Research Objective:}

The purpose of using Blockchain in the agriculture system is to solve many problems in the agriculture sector. Its purpose is to create a good, reliable, high quality, safe, and efficient agricultural system. In agriculture, an increasing number of problems related to the risks of impurity needs immediate food safety that serves as a quality management tool to ensure satisfactory crop safety and require real traceability.

\section{Socio-Economic Importance/Benefits:}

Blockchain is applied to prove how such a system increases clarity and automates connections in the agriculture sector. Blockchain is used to evaluate the accuracy of the system, simulate realworld applications, and compare the result to the output from the main office for agriculture. A Blockchain is a developing tool that allows universal financial transaction between unreliable dispersed parties, and there is no need for intermediaries.

\section{Literature Review:}

(Xiong F. et. al, 2019) explains that traditional building supply chains due to information intermediaries provide additional time, costs, and information waste. Blockchain, a regionalized, 
irreversible structure, can provide invincibility, exclusivity, and obscurity to trading winds. Therefore, here propose a Blockchain building supply chain model to reduce restrictions in oldstyle areas. However, payment security at Blockchain is a noun asset in accounts that must be protected. The discovery or alteration of records in Blockchain does not rise due to the loss of private keys due to root and block headers, false payments can be made and pin attackers can be made on all tokens in the exact header accounts detached. Current methods for privacy guards include basic biometric signature systems, important private index designs, and post-ration schemes. Therefore no one saves them as a private element. So, therefore, described a remote procedure to the safety of private keys in Blockchain (Xiong F et. al, 2019).

The limitations given in classic Blockchain in operation in the supply chain of the large manufacturing enterprise, they built a consistent data storage tool and access to Blockchain. The economic value was analyzed from aspects of the speed of response, the truth of supply, unity of cooperation, economic interaction of basic business price, quality supply, and Price supply (Fu Y \& Zhu J, 2019).

This study will deliver a complex estimation and structure for the development of Blockchain in the agriculture sector and support the expansion of Blockchain applied research in a specific region. The idea of applying Blockchain in shared risk management created the Blockchain standard and the analytical position of Blockchain applications in the academic, industry and trade loops of large manufacturing enterprises to build the system structure, as a smart contractual instrument under the secure, case, information storage instrument, and information access instrument Blockchain the operating procedure of the large chain of manufacturing enterprises, due to the selective irregularity of the selective information (Lin W. et al, 2020)

Blockchain is a regional accounting and information storage technology, and with Blockchain, we can secure the potential scam problem for commercial enterprise topics. We provide a more accurate database for all business plane divisions and make cluster decisions (Fu Y. and Zhu J., 2019).

(Kamilaris A. et. al, 2019) describe that system will protect against all attacks of secret distribution algorithms and attacks on paper-based network procedures. Also, the collaborative actions of the area units have been established for improvement and actual experiments show that point prices 
are not noticed in our system since the size orders are in retrospect of milliseconds. The system will be used in any universal situations where it is necessary to force personal keys to be safe. Blockchain is evolving in digital engineering allowing for all-powerful financial deals among an unrestricted distribution company, without the need for an intermediary such as a bank. This research explores the impact of Blockchain within the agricultural system which defines current development and advantages and defines all insinuations, tasks, and hurdles in a significant vision on the development of these plans. Our finding of blockchain in the agriculture system shows a transparent food system with many current initiatives in several crops and food systems but still different complications and challenges that delay the use of a broader reputation among agriculturalists and the system. These tasks concern a technical, instructional, policy, and supervisory agenda.

There is a globalized manufacturing and agricultural sharing on the protection, value, and authentication of significant new principles in the agriculture system. The rising number of problems of food protection and toxic waste risks has identified that essential traceability results have proven to be an important quality management tool that guarantees traceable crop protection in the agriculture supply chain (Salah K et. al., 2019). Blockchain is capable of achieving high yields for traceability of products in the agriculture sector. Blockchain is already using many plans and initiatives and we expect to find a trusted verification environment to create clearer and workable food manufacturing and sharing.

They need to fund new investments and improvements. For example in education and preparation, to provide an indication and to consider the potential margins of blockchain. The potential shift between the use of blockchain by the government noticing the government and its respective divisions always detect and perceive the actual "pain points" and then speak them(Salah K et. al., 2019)

According to (Mao D. et. al., 2019) describe that the Blockchain consortium model was leveraged on an automatic dealing scheme, where food can be listed separately from a trusted third party. It benefits from merchant incomes. Safety analysis has shown that the projected technique promotes the safety and confidentiality of deals. Arithmetical analysis confirms the strength of the scheme in maximizing income for traders with a minimal computational price. Importantly, the basic concept of Blockchain still has problems with computing resources, deal price, block speed, and 
interoperability (Motta G.A et,al 2020). The features of these research and units may be quite generic in character and reliable and decentralized traceability can be applied to any product or product within the agricultural supply chain Blockchain has key dares linked to sustainability, control, identity registration, confidentiality, values, and laws. As future work, we usually decide to consider many key challenges and give outcomes and then address them (Mao D. et. al., 2019).

(Batubara F. et. al., 2018) explains that many issues are confronting traditional food trading platforms, such as the rapid detection of trade goods and protecting the consistency of transaction information. With the rapid emergence of e-commerce, food trade has shifted to the online arena. Blockchain has changed many businesses due to their robustness, regionalization, and eventual reliability. This paper proposes a new food dealing system by using blockchain to develop trust and safety problems in transactions. It uses Blockchain conglomerate technology to arrange consent and certification for various parts of a food dealing system.

\section{Research Methodology:}

The main purpose of this research is to give a summary of the research and determine the research type, result, and quantity available with this. The following steps are used for SMS. 


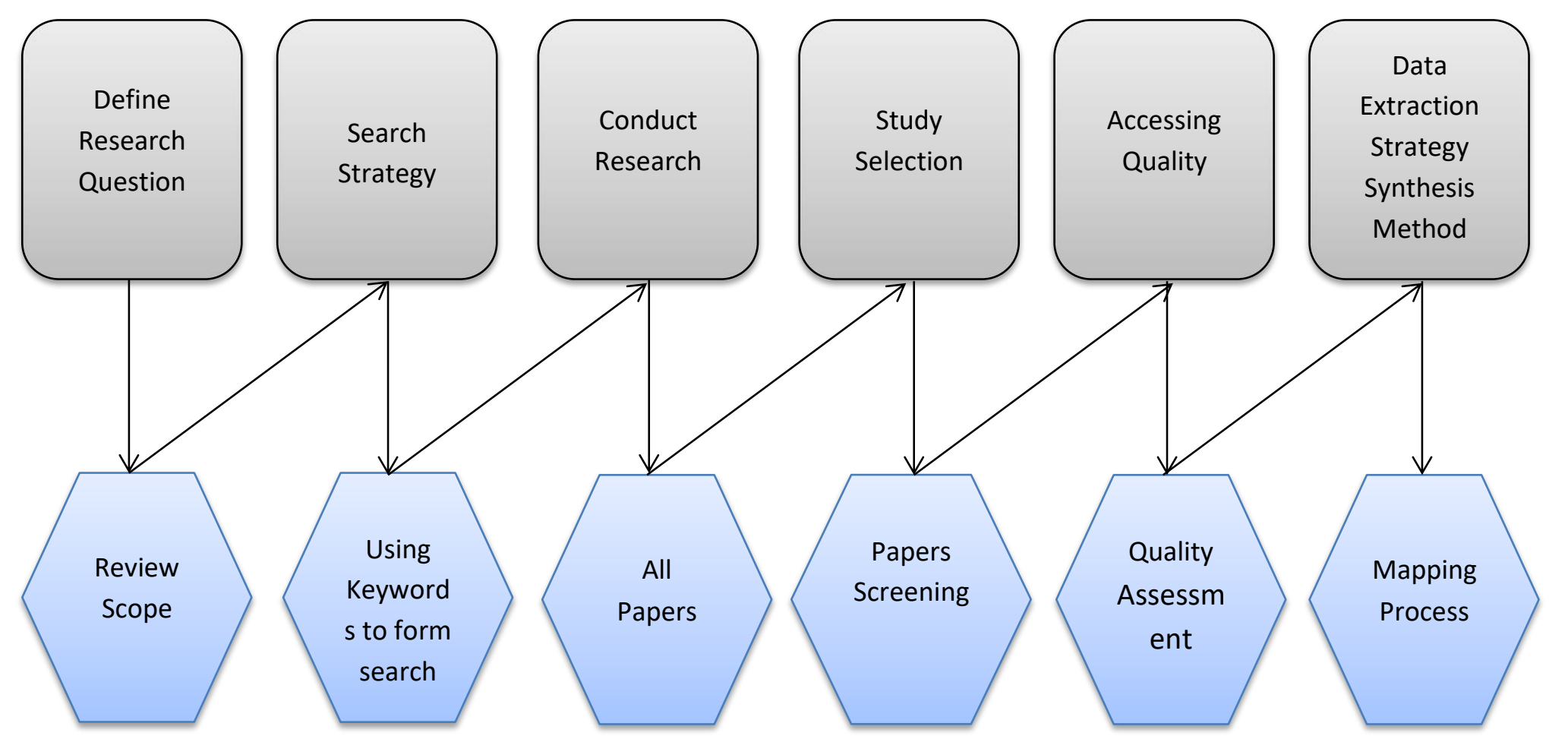

\section{Steps.}

1) Identify research Questions

2) Search Strategy

3) Conduct research

4) Study Selection

5) Accessing Quality

6) Synthesis Method

7) Document result and outcomes

\section{Identify research questions}

The objective of this study is to raise awareness in the solutions designed to handle agriculture issues to gain a detailed insight into this issue and this systematic mapping study addresses the questions. The questions will assist us to classify existing Blockchain researchers which use in agriculture and also define the further research areas in this field. 


\section{Search Strategy}

In this step find keywords to form a query string. The keywords which we used to find journals are following

- Blockchain

- Agriculture

\section{Conduct Research}

The area in which some relevant authors research has been published. The sources which we used to find journals are the following.

- IEEE explore

- ACM digital library

- Science direct

- Springer

- Elsevier

\section{Study selection}

Study selection is the process of selecting studies which is included or which is excluded. The purpose of the inclusion process was to categorize the journals most relevant to the purpose of this study. The study is in English. Peer-reviewed articles are included. Papers published until 2015 are included.

The exclusion criteria included the studies which are not written in English. The studies which are not Peer-reviewed. Short papers (less than four pages). Papers that are not focused on Blockchain and agriculture system. Papers not presenting a general focus Blockchain and agriculture system. Papers about requirements Blockchain not using in the agriculture system.

\section{Quality assessment}

To improve our study, we designed a questionnaire that assesses the quality of the selected papers. Both authors retrieved questions/answers conducted by the studies. Blockchain guiding principle acclaim a consideration of the usefulness of the agricultural system, conduct from these points:

- Innovation 
- Security

- Transparency

\section{Synthesis method \& Data extraction strategy}

A method that is used to combine results from various studies and make a quantitative estimate of the whole outcome of a given innovation on a result.

\section{Results}

- The new food dealing system is present by the Blockchain to solve problems like security, safety, transparency in transactions.

- Blockchain is used to fixed agreement and approval for many portions in a food transaction.

- Blockchain has achieved great scientific and commercial interests for farmers.

- Blockchain is helpful to the farmers who can solve many issues like inefficiency, food fraud, food traceability, and safety recalls in the food system.

- By using Blockchain efficiency is improved by eliminating different steps in the communication process then orders are complete accurately and faster.

- In agriculture, an increasing number of problems linked to the risks of impurity and immediate food safety are needed to achieve a better traceability outcome as an essential quality controlling device to ensure the acceptable safety of crops.

- Blockchain has changed many businesses because of its robustness, secure environment, and reliability.

- In the future, investment in Blockchain continues to grow, and innovative applications are rapidly developed.

\section{References}


1. Batubara, F. R., Ubacht, J., \& Janssen, M. (2018). Challenges of blockchain technology adoption for e-government: A systematic literature review. ACM International Conference Proceeding Series.

2. Caro, M. P., Ali, M. S., Vecchio, M., \& Giaffreda, R. (2018). Blockchain-based traceability in Agri-Food supply chain management: A practical implementation. 2018 IoT Vertical and Topical Summit on Agriculture - Tuscany, IOT Tuscany 2018, 1-4.

3. Fu, Y., \& Zhu, J. (2019). Big Production Enterprise Supply Chain Endogenous Risk Management Based on Blockchain. IEEE Access, 7, 15310-15319.

4. Kamilaris, A., Fonts, A., \& Prenafeta-Boldv́, F. X. (2019). The rise of Blockchain technology in agriculture and food supply chains. Trends in Food Science and Technology, $91,640-652$

5. Kitchenham, B., Mendes, E., Travassos, G.H. (2007) A Systematic Review of Cross- vs. Within-Company Cost Estimation Studies, IEEE Trans on SE, 33 (5), pp 316-329.

6. Lin, Y.-P., Chang, T.-K., Fan, C., Anthony, J., Petway, J., Lien, W.-Y., ... Ho, Y.-F. (2017). Applications of Information and Communication Technology for Improvements of Water and Soil Monitoring and Assessments in Agricultural Areas-A Case Study in the Taoyuan Irrigation District. Environments, 4(1), 6.

7. Lin, W., Huang, X., Fang, H., Wang, V., Hua, Y., Wang, J., Yin, H., Yi, D., \& Yau, L. (2020). Blockchain technology in current agricultural systems: from techniques to applications. IEEE Access, August, 1-1.

8. Mao, D., Hao, Z., Wang, F., \& Li, H. (2019). Novel Automatic Food Trading System Using Consortium Blockchain. Arabian Journal for Science and Engineering, 44(4), 3439-3455.

9. Motta, G. A., Tekinerdogan, B., \& Athanasiadis, I. N. (2020). Blockchain Applications in the Agri-Food Domain: The First Wave. Frontiers in Blockchain, 3(February), 1-13.

10. Ouhbi, S., Idri, A., Fernández-Alemán, J. L., \& Toval, A. (2015). Requirements engineering education: a systematic mapping study. Requirements Engineering, 20(2), 119-138 
11. Salah, K., Nizamuddin, N., Jayaraman, R., \& Omar, M. (2019). Blockchain-Based Soybean Traceability in Agricultural Supply Chain. IEEE Access, 7, 73295-73305.

12. Tian, F. (2010). An Agrifood Supply Chain Traceability System for China Based on RFID \& Blockchain Technology.

13. Xiong, F., Xiao, R., Ren, W., Zheng, R., \& Jiang, J. (2019). A Key Protection Scheme Based on Secret Sharing for Blockchain-Based Construction Supply Chain System. IEEE Access, 7, 126773-126786

14. Yang, H., Xiong, S., Frimpong, S. A., \& Zhang, M. (2020). A consortium blockchainbased agricultural machinery scheduling system. Sensors (Switzerland), 20(9). 
